\title{
CONFÉRENCE
}

\section{Reconstruction osseuse planifiée et pose simultanée d'implants}

\author{
Andrew Dawood \\ Université de Londres \\ andrewdawood@hotmail.com
}

L'imagerie 3D et les technologies d'impression tridimensionnelle ont complètement modifié l'approche des traitements complexes autant sur le versant chirurgical que prothétique.

Les progrès spectaculaires de ces imprimantes 3D font qu'il est envisageable dès aujourd'hui d'en disposer au fauteuil.

Les industriels proposent des dispositifs qui vont de solutions grand public type Maker Boat accessible sur Internet pour fabriquer de petites pièces en résine, à des solutions professionnelles beaucoup plus complexes pouvant aller jusqu'à la fabrication de prothèse. L'impression produit des pièces pour une analyse plus simple des situations anatomiques et prothétiques complexes.

De cette analyse découle une stratégie facilitée par la production de guides. L'exemple le plus ancien est bien évidemment la production de guides chirurgicaux proposée pour la première fois il y a une vingtaine d'années par une équipe française.

Depuis, plusieurs équipes ont développé différentes approches, mécaniques ou par impression type stéréolithographique, non pas uniquement pour la pose d'implants mais également pour les tracés d'ostéotomies. Les modèles stéréolithographiques nous permettent, et c'est leur spécificité, de simuler en pré-opératoire les greffons osseux à reporter sur le site chirurgical pour minimiser l'importance des incisions, la perte osseuse sur le site de prélèvement mais aussi préparer les solutions prothétiques dans la perspective d'une parfaite prédictibilité des résultats. 